\title{
Uso inadecuado de medicamentos en adultos mayores
}

\author{
Teodoro J Oscanoa ${ }^{1,2}$
}

Resumen Objetivo: Determinar la prevalencia de la prescripción potencialmente inadecuada y las interacciones fármaco-enfermedad (definido por los criterios de Beers) en pacientes hospitalizados y los factores asociados a su uso. Material y Métodos: Ingresó al estudio una muestra de 500 pacientes (media edad: 75 años) hospitalizados entre junio 2002 y junio 2003. Se consideró la medicación prescrita antes (medicación habitual), durante y al alta del hospital. Resultados: La prevalencia de prescripción de al menos un medicamento inapropiado al ingreso, durante la hospitalización y al alta fue de $12,4 \%, 3,4 \%$ y 2,1\%, respectivamente. Los medicamentos más frecuentemente implicados al ingreso hospitalario fueron diazepám, digoxina (dosis $>0,125 \mathrm{mg} /$ día), hierro (dosis superiores a $325 \mathrm{mg} /$ día), clorfeniramina y amitriptilina. La interacción droga-enfermedad potencialmente adversa al ingreso, durante el internamiento y al alta fue de 13,4\%, 5,4\% y 4,2\%, respectivamente. Las variables número de fármacos al ingreso y el número de enfermedades y el ítem dolor del puntaje COOP/WONCA fueron estadísticamente significativas. Conclusiones: El estudio revela la existencia de prescripción de medicación potencialmente inadecuada o de interacción droga-enfermedad potencialmente adversa en pacientes hospitalizados en nuestro medio. La polifarmacia, polipatología y la presencia de dolor crónico se asociaron significativamente con la prescripción potencialmente inadecuada.

Palabras clave Drogas, efectos adversos; prescripción de medicamentos; farmacología, efectos adversos; anciano.

\section{Inadequate medication use in older adults}

\section{Abstract}

Objectives: To determine the prevalence of potentially inappropriate medication use and adverse drug-disease (defined by the Beers' criteria) among hospitalized older adults and to identify predictors of this use. Material and Methods: A sample of 500 patients (mean age 75 years) admitted to the hospital between June 2002 and June 2003 was included in this analysis. Medication before, during or at discharge from hospitalization was considered for the present study. Results: On admission to the hospital 12,4\% of the patients was taking a potentially inappropriate medication, 3,4\% was given one medicine in the hospital, and $2,1 \%$ was prescribed one medication upon discharge. The most frequently potentially inappropriate medications prescribed on admission were diazepam, digoxin (>0,125

\footnotetext{
1 Servicio de Geriatría, Hospital Nacional Guillermo Almenara Irigoyen. Lima Perú.

2 Facultad de Medicina, Universidad Mayor de San Marcos. Lima, Perú.
}

mg/day), iron supplements (>325 mg), chlorpheniramine, and amitriptyline. The prevalence of potentially adverse drug-disease interactions was $13,4 \%$ on admission, $5,4 \%$ during hospital stay and 4,2\% on discharge. Statistical analysis with COOP/WONCA score showed that admission number of medications, number of diseases and pain were significantly associated with use of inappropriate medications. Conclusions: Our study revealed the existence of potentially inappropriate medications use among older adults in a Lima, Peru hospital inpatients. Polypharmacy, comorbidity and chronic pain were significantly associated with inappropriate medications use.

Key words: Drugs, adverse effects; pharmacology, adverse effects; prescriptions, drug; aged.

\section{INTRODUCCIÓN}

Las enfermedades inducidas por medicamentos en los ancianos están cobrando importancia 
debido a que la población de adultos mayores ha aumentado. Las investigaciones sugieren que la terapia farmacológica inadecuada o inapropiada es un importante factor de riesgo para estas enfermedades relacionadas con los fármacos. Se define medicación inadecuada cuando el riesgo sobrepasa el beneficio $\left({ }^{1}\right)$. La tasa de riesgo / beneficio de algunos medicamentos resulta afectada por los cambios en la farmacocinética y farmacodinámica relacionados con los procesos de envejecimiento. Prescribir un fármaco inadecuado a la población anciana lo expone a un riesgo de morbilidad y mortalidad. Por lo tanto, es importante que los medicamentos inapropiados sean identificados, sus patrones de uso actuales entendidos y los errores de prescripción corregidos en el futuro.

Las reacciones adversas medicamentosas (RAM) son las consecuencias más serias de la prescripción inadecuada. Las RAM cuando no son reconocidas pueden a su vez inducir a la prescripción de adicionales medicamentos con detrimento de la calidad de vida. Consecuentemente las RAM mencionadas pueden resultar en hospitalizaciones innecesarias y un incremento en la morbilidad y mortalidad. Un reciente metaanálisis ${ }^{2}$ ) estimó que 1,5 millones ó 4,7\% de todas las hospitalizaciones fueron debidas a RAM. Estudios limitados a ancianos encontraron frecuencias tan altas como $17 \%$. La manera más efectiva de prevenir los RAM en ancianos es reduciendo el número de prescripciones inadecuadas.

En 1991 Beers y col. desarrollaron los Criterios Explícitos de Medicación Inadecuada en el adulto mayor, que luego fueron actualizados en 1997 mediante la técnica del consenso de expertos y de conceptos de medicina basada en evidencias $\left({ }^{3}\right)$. Ellos incluyen fármacos que deberían ser evitados absolutamente en ancianos y otras drogas cuyas dosis, frecuencia de administración o duración no deberían de exceder ciertos límites. Varios de estos fármacos fueron considerados inadecuados debido a que existen alternativas más seguras. Por ejemplo, las benzodiazepinas de actividad corta tienen un mejor perfil de seguridad que las benzodiazepinas de larga duración, meprobamato y barbitúricos de acción corta. Otros agentes fueron considerados inapropiados debido a que sus efectos son extremadamente problemáticos para el geronte. Tales agentes incluyen amitriptilina (alto grado de hipotensión ortostática y efectos anticolinérgicos), algunos antihipertensivos (con efectos sobre el sistema nerviosos central (SNC), como metildopa y reserpina), antiinflamatorios no esteroideos -como indometacina y fenilbutazona (efectos sobre el SNC y toxicidad sobre la médula ósea, respectivamente), clorpropamida (vida media prolongada y posibilidad de síndrome de secreción inadecuada de hormona antidiurética), propoxifeno y pentazocina (efectos sobre el SNC y toxicidad cardiaca), dipiridamol (efectos sobre el SNC), relajantes musculares (efectos sobre el SNC) y trimetobenzamida (mareos, diarrea, rash y síntomas extrapiramidales). Algunos agentes fueron considerados inapropiados por su cuestionable eficacia, entre estos medicamentos están proproxifeno, pentazocina, ciclandenato, isoxsuprina, dipyridamol y trimetobenzamida $\left({ }^{3,4}\right)$.

Los criterios de uso inadecuado están referidos también al uso de medicamentos que exceden la dosis máxima o que sobrepasan la duración usual y que por ello causan alta frecuencia de reacciones adversas y son de cuestionable necesidad $^{5}$. Por ejemplo, la terapia con hierro no debe exceder de $325 \mathrm{mg} /$ día; si las dosis son mayores, no son absorbidas, pero incrementan sus reacciones adversas $\left({ }^{3}\right)$. Por otro lado, si el uso es único o muy infrecuente también es considerado inapropiado, dado que no se consigue el efecto deseado.

Beers y col también identificaron los fármacos que deben ser evitados en pacientes con antecedentes de una de las 14 enfermedades, a lo que denominó interacción droga-enfermedad $\left({ }^{5}\right)$. Los integrantes del grupo de Consenso también identificaron 4 interacciones medicamento-medicamento, que deben ser 
evitados (vgr. Warfarina, ya sea con aspirina, AINEs (antiinflamatorios no esteroideos), dipiridamol o ticlopidina) y tres medicamentos o clases de medicamentos con dosis específicas recomendadas en ancianos: 1) digoxina, cuya dosis no debe exceder $0,125 \mathrm{mg} /$ día, excepto en casos de arritmia auricular; 2) benzodiazepinas de vida media corta que no deberían exceder de una dosis total diaria (alprazolam $>2 \mathrm{mg}$, lorazepam $>3 \mathrm{mg}$, oxazepam $>60 \mathrm{mg}$, temazepam $>15 \mathrm{mg}$, triazolam $>0,25$, zolpidem $>5 \mathrm{mg}$ ); y, 3) sulfato ferroso, cuya dosis no debe exceder de $325 \mathrm{mg} /$ día $\left(^{6}\right)$.

Stuck y col. $\left(^{7}\right)$ modificaron la lista original de Beers aplicándola a pacientes atendidos en sus domicilios. Esta lista es denominada Criterios de Beers Modificado e incluye antiespasmódicos gastrointestinales, pero excluye metildopa y propanolol. Spore y col. $\left.{ }^{8}\right)$ aplicaron los criterios usados por Stuck para examinar prescripción inadecuada en hospitales de día geriátricos con una lista más amplia de benzodiazepinas de actividad prolongada. La lista de 20 medicamentos que deben ser evitados en el anciano fue el foco de estudios por el Government Accounting Office (GAO) en EE UU, comprometiendo a beneficiarios del Medicare. Willcox y col. $\left({ }^{4}\right)$ extendieron a ancianos de atención domiciliaria y Aparasu y Fliginer $\left({ }^{9}\right)$ a pacientes de consultorio externos. Finalmente, se ha realizado estudios sobre prescripción inadecuada de fármacos en ancianos atendidos en salas de emergencia de hospitales de EE UU empleando los criterios de Beers $\left({ }^{10}\right)$.

El presente estudio tiene el objetivo de determinar la prevalencia de prescripción inadecuada de fármacos en la población anciana, antes, durante y al alta del Hospital Nacional Guillermo Almenara Irigoyen, en Lima, Perú.

\section{MATERIAL Y MÉTODOS}

El presente es un estudio prospectivo, descriptivo, transversal y observacional. El estudio fue realizado en pacientes hospitalizados del Hospital Nacional Guillermo Almenara Irigoyen, en Lima, Perú. El hospital mencionado es de alta complejidad y cuenta con 1200 camas. Se obtuvo una muestra de 500 pacientes mayores de 60 años hospitalizados entre junio 2002 y junio 2003. La población de pacientes hospitalizados incluyó aproximadamente el $60 \%$ de pacientes del servicio de Geriatría (hospitalizados entre junio y octubre de 2002 y durante el mes de enero de 2003) y el restante $40 \%$ de otros servicios, entre los cuales estuvieron Nefrología, Neumología, Ortopedia y Traumatología, Emergencia, Oncología, Reumatología y un servicio de Medicina Interna, estos últimos hospitalizados entre febrero y junio 2003. Los servicios mencionados, con excepción del servicio de Geriatría y Emergencia, fueron incluidos al ser sorteados de la totalidad de servicios de hospitalización del Hospital Almenara.

Se elaboró un cuestionario especial para el estudio, que incluyó datos demográficos, como edad, sexo, estado civil, raza y grado de instrucción. Otras variables evaluadas fueron el número de hospitalizaciones en los 12 meses previos, número de fármacos al ingreso (medicación habitual o previa a la hospitalización), durante la hospitalización y al alta (o fallecimiento) del paciente. Para la evaluación del estado mental, se empleó el miniexamen cognoscitivo Lobo $\left({ }^{11}\right)$; para la evaluación de las actividades de la vida diaria (AVD) se usó el índice de Katz $\left({ }^{12}\right)$ y el puntaje de COOP/WONCA para calificar la calidad de vida y evaluación funcional de la salud $\left({ }^{13}\right)$. Si el paciente presentaba trastorno del sensorio o un puntaje de miniexamen cognoscitivo de Lobo menor de 17, el puntaje de COOP/WONCA se hizo a través del cuidador y, en ausencia de éste, la calificación fue hecha por el médico encuestador.

Se usó los criterios de Beers, de 1997, sobre prescripción inadecuada de medicamentos $\left(^{5}\right)$ Estos criterios explícitos fueron desarrollados en EE UU por un método Delphi modificado con 
un panel de seis reconocidos expertos en geriatría, farmacología clínica, fármaco epidemiología, farmacia clínica y psicofármacología.

Según los criterios de Beers, son drogas que deberían ser evitadas por ser inefectivas o tienen un riesgo innecesariamente alto para los adultos mayores, y también las drogas que son apropiadas para su uso en ancianos sólo a ciertas dosis, frecuencia o duración de terapias $\left({ }^{5}\right)$.

Los criterios de Beers también incluyen drogas que no deberían ser usadas en ancianos que se conoce que tienen determinadas condiciones médicas. Estos criterios de combinaciones potencialmente adversas de droga-enfermedad cubren 15 condiciones y enfermedades $\left({ }^{5}\right)$. Por ejemplo, el uso de beta bloqueadores en un paciente con asma puede empeorar la función respiratoria. Se identificó estas condiciones en los pacientes al examinarlos, usando los datos de la historia clínica y revisando las epicrisis.

La aprobación del presente estudio fue realizada bajo criterios éticos de la Institución EsSalud, la Declaración de Helsinki de 1975, enmendada en 1983. La detección de medicación potencialmente inadecuada e interacción drogaenfermedad potencialmente adversa fue seguida de comunicación al médico tratante para la suspensión del fármaco detectado como inadecuado; excepcionalmente, al alta siguieron con el mismo medicamento, especialmente cuando dicho fármaco tenía indicación diferente de lo estipulado en los criterios de Brees $\left({ }^{5}\right)$ (ejemplo, amitriptilina como terapia para neuropatía diabética).

Se registró los fármacos prescritos potencialmente inadecuados así como también las interacciones adversas fármaco-enfermedad. También se anotó los fármacos que estaba tomando el paciente antes de ser hospitalizado (medicación habitual), durante la hospitalización y al momento de ser dado de alta; en cada uno de los casos se correlacionó si la medicación prescrita era potencialmente inadecuada o presentaba interacción adversa fármacoenfermedad. Las variables sociodemográficas analizadas fueron edad, sexo, raza, grado de instrucción, estado civil. Las variables clínicas analizadas incluyeron el número de medicamentos, número de enfermedades, índice de Katz y número de admisiones previas en los últimos 12 meses. En todos los casos se determinó el puntaje de COOP/WONCA, por autoevaluación o a través del cuidador o el médico encuestador si ello era necesario. La edad fue categorizada en 60-69 años, 70-79 años y 80 ó más años. El grado de instrucción fue categorizado en analfabetos, primaria (1-6 años de estudio), secundaria (7 a 12 años) y superior (más de 12 años). Para establecer las diferencias estadísticas de las variables en relación con el grupo con y sin prescripción inadecuada, se utilizó la prueba del chi cuadrado para variables categóricas y la prueba de la suma de rangos de Wilcoxon para variables continuas.

\section{RESULTADOS}

El total de pacientes hospitalizados estudiados fueron 500. Ingresaron al estudio los pacientes hospitalizados en el servicio de Geriatría durante los meses junio a octubre de 2002 y enero de 2003; en esos periodos ingresaron un total de 322 pacientes, habiéndose incluido en el estudio a 300; 22 casos fueron excluidos por no tener información de medicamentos antes de su hospitalización o el paciente / cuidador no las recordaba. Durante los meses de febrero y junio 2003 ingresaron al estudio 205 pacientes adultos mayores procedentes de los servicios de Nefrología, Neumología, Ortopedia y Traumatología, Emergencia, Oncología, Reumatología y un servicio de Medicina Interna. En este último grupo se excluyó a 5, por los mismos motivos del primer grupo. La mortalidad intrahospitalaria de los pacientes estudiados fue $8,3 \%$ (26 fallecidos).

La media de edad fue 75,2 años (rango: 60 a 100 años), $52 \%$ del sexo masculino, $58 \%$ 
casados y $50 \%$ con nivel de instrucción primaria (Tabla 1). La media del número de enfermedades registrada fue 4,1 , la media de deficiencias en las actividades de la vida diaria (alimentación, continencia, transferencia, uso del retrete, vestir, baño) fue 2,6 y $72 \%$ tuvo por lo menos una deficiencia en las actividades de la vida diaria (AVD). La media del número de fármacos al ingreso (medicación habitual antes de ser hospitalizado), durante hospitalización $\mathrm{y}$ al alta fueron $3,2,4,5$ y 2,3, respectivamente (Tabla 2).

Se detectó la prescripción de al menos un medicamento inapropiado al ingreso, durante la hospitalización y al alta en $12,4 \%, 3,4 \%$ y $2,2 \%$, respectivamente (Tabla 3). Los cinco medicamentos más frecuentemente prescritos inadecuadamente fueron diazepam, digoxina (dosis superiores a $0,125 \mathrm{mg} /$ día), hierro (dosis superiores a $325 \mathrm{mg} /$ día), clorfeniramina y amitriptilina. Durante la hospitalización, los

Tabla 1. Características demográficas de la población estudiada.

\begin{tabular}{lc}
\hline Características & Valores \\
\hline Edad (años, media \pm DE) & $75,2 \pm 8,8$ \\
Sexo & (Rango: $60-100$ años) \\
Masculino & $52 \%$ \\
Femenino & $48 \%$ \\
Estado civil & \\
Casado & $58 \%$ \\
Viudo/a & $31 \%$ \\
Soltero & $6 \%$ \\
Divorciado & $4 \%$ \\
Conviviente & $1 \%$ \\
Grado de instrucción & \\
Analfabeto & \\
Primaria & $5 \%$ \\
Secundaria & $50 \%$ \\
Superior & $30 \%$ \\
Raza & $15 \%$ \\
Mestiza & \\
Blanca & $95 \%$ \\
Otros & $4 \%$ \\
\end{tabular}

DE: Desviación estándar.
Tabla 2. Características clínicas de la población estudiada.

\begin{tabular}{|c|c|}
\hline Características clínicas & Valores \\
\hline Número de fármacos al ingreso (media $\pm \mathrm{DE}$ ) & $3,2 \pm 2,1$ \\
\hline $\begin{array}{l}\text { Número de fármacos durante hospitalización } \\
\text { (media+DE) }\end{array}$ & $4.5 \pm 2,1$ \\
\hline 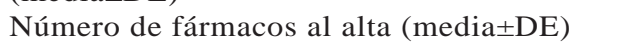 & $2,3 \pm 1,5$ \\
\hline Número de enfermedades (media \pm DE) & $4,1 \pm 1,1$ \\
\hline Número de hospitalizaciones en & \\
\hline los 12 meses previos (media $\pm \mathrm{DE}$ ) & $1,2 \pm 1,0$ \\
\hline $\begin{array}{l}\text { Número de deficiencias en las actividades } \\
\text { de la vida diaria (alimentación, continencia, } \\
\text { transferencia, uso del retrete, vestir, baño) }\end{array}$ & \\
\hline$($ media $\pm \mathrm{DE})$ & $2,6 \pm 2,3$ \\
\hline Actividades de la vida diaria: Katz & \\
\hline A & $28 \%$ \\
\hline $\mathrm{B}$ & $12 \%$ \\
\hline $\mathrm{C}$ & $14 \%$ \\
\hline $\mathrm{D}$ & $9 \%$ \\
\hline $\mathrm{E}$ & $7 \%$ \\
\hline $\mathrm{F}$ & $11 \%$ \\
\hline G & $19 \%$ \\
\hline $\begin{array}{l}\text { Calidad de vida y evaluación funcional } \\
\text { de la salud: puntaje de COOP/WONCA }\end{array}$ & \\
\hline Capacidad física & $3,8 \pm 1,0$ \\
\hline Sentimientos & $3,3 \pm 0,9$ \\
\hline Actividades cotidianas & $3,7 \pm 1,0$ \\
\hline Actividades sociales & $3,8 \pm 1,0$ \\
\hline Cambio en el estado social & $3,7 \pm 1,0$ \\
\hline Estado de salud & $4,0 \pm 0,8$ \\
\hline Dolor & $2,6 \pm 1,2$ \\
\hline Apoyo social & $2,8 \pm 1,0$ \\
\hline Calidad de vida & $3,8 \pm 1,0$ \\
\hline
\end{tabular}

DE: Desviación estándar.

fármacos más frecuentes fueron diazepan e hioscina, aunque la prevalencia de prescripción inadecuada fue mucho menor.

Se observó interacción droga-enfermedad potencialmente adversa al ingreso, durante el internamiento y al alta en porcentajes de $13,4 \%$, $5,4 \%$ y $4,2 \%$, respectivamente. Las cinco interacciones droga-enfermedad potencialmente adversa más frecuentes fueron úlcera péptica con antiinflamatorios no esteroideos, enfermedad pulmonar obstructiva crónica con beta bloqueadores y sedantes, diabetes mellitus y betabloqueadores (en pacientes con 
Tabla 3. Frecuencia de prescripción potencialmente inadecuada*.

\begin{tabular}{|c|c|c|c|}
\hline Fármaco & $\mathrm{Al}$ ingreso & $\begin{array}{c}\text { Durante la } \\
\text { hospitalización }\end{array}$ & Al alta \\
\hline Diazepam & 16 & 5 & \\
\hline Digoxina & 16 & 3 & 2 \\
\hline Hierro & 7 & & \\
\hline Clorfeniramina & 7 & & \\
\hline Amitriptilina & 5 & 1 & 3 \\
\hline Clorpropamida & 3 & & \\
\hline Metildopa & 3 & & \\
\hline Alprazolam & 2 & 3 & 6 \\
\hline Flunitrazepam & 1 & & \\
\hline Mesilato & & & \\
\hline de codergocrina & 1 & & \\
\hline Hioscina & 1 & 5 & \\
\hline Total (\%) & $62(12,4)$ & $17(3,4)$ & $11(2,2)$ \\
\hline
\end{tabular}

* Según los criterios de Beers sobre prescripción potencialmente inadecuada $\left({ }^{5}\right)$.

Algunos pacientes tuvieron más de una prescripción inadecuada.

hipoglicemiantes orales) y, finalmente, hipertrofia benigna de próstata con amitriptilina y clorfeniramina (Tabla 4).

La prescripción de medicación potencialmente inadecuada o de interacción droga-enfermedad potencialmente adversa al ingreso al área de hospitalización fue relacionada con las siguientes variables: número de fármacos al ingreso, número de enfermedades y puntaje de COOP/WONCA para calificar la calidad de vida y evaluación funcional de la salud. Las variables que no resultaron ser estadísticamente significativas fueron: edad, sexo, estado civil, raza, escolaridad, índice de Katz, número de hospitalizaciones 12 meses antes del internamiento cuando ingresó al estudio y mortalidad intrahospitalaria. Las variables número de fármacos al ingreso y el número de enfermedades y el ítem dolor del puntaje de COOP/WONCA fueron estadísticamente significativas (Tablas 5 y 6). Colateralmente, se evidenció que el antecedente al ingreso de prescripción de medicación potencialmente inadecuada o interacción droga-enfermedad potencialmente adversa tuvo una frecuencia muy significativa de sufrir una reacción adversa medicamentosa (riesgo relativo 4,11 intervalo de confianza 2,3-7,2 y una $p<0,001$ ) comparado

Tabla 4. Frecuencia de interacciones fármacoenfermedad potencialmente adversa* .

\begin{tabular}{|c|c|c|c|}
\hline $\begin{array}{l}\text { Enfermedad } \\
\text { y fármaco }\end{array}$ & $\mathrm{Al}$ ingreso & $\begin{array}{c}\text { Durante la } \\
\text { hospitalización }\end{array}$ & Al alta \\
\hline
\end{tabular}

\begin{tabular}{lc}
\hline Enfermedad úlcero péptica \\
Diclofenaco & 13 \\
Ibuprofeno & 7 \\
Aspirina & 3 \\
Piroxicam & 1
\end{tabular}

Hipertrofia benigna de próstata

Clorfeniramina 5

Amitriptilina 1

Diabetes mellitus

Propanolol 3

Atenolol 5

Síncopes o caídas

Atenolol 5

$\begin{array}{llll}\text { Propanolol } & 1 & 5 & 4\end{array}$

Enfermedad pulmonar obstructiva crónica

$\begin{array}{llll}\text { Alprazolam } & 3 & 2 & 2\end{array}$

Atenolol 3

Diazepam 1

$\begin{array}{llll}\text { Propranolol } & 3 & 1 & 2\end{array}$

Estreñimiento

$\begin{array}{llll}\text { Morfina } & 3 & 3 & 4\end{array}$

Codeína

1

Hioscina

Enfermedad vascular periférica

Atenolol

3

2

$3-3$

Trastornos de coagulación, limitado

a aquellos con anticoagulación

Ibuprofeno 1

Piroxicam 1

Diclofenaco 2

Asma bronquial

Atenolol

Propanolol

1

Total (\%)

$67(13,4 \%)$

$27(5,4 \%) \quad 21(4,2 \%)$

\footnotetext{
* Según los criterios de Beers sobre prescripción potencialmente inadecuada $\left({ }^{5}\right)$.

Algunos pacientes tuvieron más de una prescripción inadecuada.
} 
Tabla 5. Prescripción potencialmente inadecuada de medicamentos o de interacciones adversas fármaco-enfermedad: relación con número de fármacos y número de enfermedades.

\begin{tabular}{cccc}
\hline Variable & $\begin{array}{c}\text { Pacientes CON } \\
\text { prescripción }\end{array}$ & $\begin{array}{c}\text { Paciente SIN } \\
\text { prescripción }\end{array}$ & Valor \\
potencialmente & potencialmente & de \\
inadecuada o & inadecuada o & $p$ \\
interacciones & interacciones & \\
$(\mathrm{n}=115)(23 \%)$ & $(\mathrm{n}=385)(77 \%)$ & \\
\hline
\end{tabular}

Número de fármacos

al ingreso (Medicación

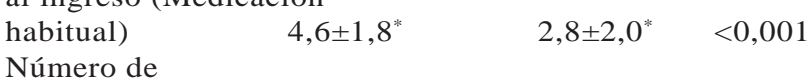

enfermedades $\quad 5,0 \pm 1,0^{*} \quad 3,9 \pm 1,0^{*} \quad<0,001$

* Media y desviación estándar.

con el grupo sin el antecedente descrito; además, en el primer grupo señalado, la reacción adversa medicamentosa fue la causa directa de la hospitalización en un $26,1 \%$ de los casos. La

Tabla 6. Prescripción potencialmente inadecuada de medicamentos o de interacciones adversas fármaco-enfermedad: estatus funcional y calidad de vida según COOP/WONCA*.

\begin{tabular}{|c|c|c|c|}
\hline $\begin{array}{l}\text { ÍItems del } \\
\text { puntaje } \\
\text { COOP/ } \\
\text { WONCA }\end{array}$ & $\begin{array}{c}\text { Pacientes CON } \\
\text { prescripción } \\
\text { potencialmente } \\
\text { inadecuada o } \\
\text { interacciones } \\
\text { (n=115) (23\%) } \\
\text { Media y DE }\end{array}$ & $\begin{array}{c}\text { Paciente SIN } \\
\text { prescripción } \\
\text { potencialmente } \\
\text { inadecuada o } \\
\text { interacciones } \\
(\mathrm{n}=385)(77 \%) \\
\text { Media y DE }\end{array}$ & $\begin{array}{c}\text { Valor } \\
\text { de } \\
p\end{array}$ \\
\hline Forma física* & $4,2 \pm 0,8$ & $3,6 \pm 0,9$ & 0,061 \\
\hline Sentimientos* & $3,6 \pm 0,8$ & $3,3 \pm 0,9$ & 0,123 \\
\hline $\begin{array}{l}\text { Actividades } \\
\text { cotidianas }^{*}\end{array}$ & $4,1 \pm 0,9$ & $3,5 \pm 0,9$ & 0,843 \\
\hline $\begin{array}{l}\text { Actividades } \\
\text { sociales }\end{array}$ & $4,3 \pm 1,0$ & $3,6 \pm 1,0$ & 0,129 \\
\hline $\begin{array}{l}\text { Cambios en el } \\
\text { estado de salud* }\end{array}$ & $4,1 \pm 1,0$ & $3,5 \pm 0,9$ & 0,760 \\
\hline Estado de salud* & $4,4 \pm 0,7$ & $3,9 \pm 0,8$ & 0,756 \\
\hline Dolor ${ }^{*}$ & $3,3 \pm 1,2$ & $2,4 \pm 1,1$ & $<0,001$ \\
\hline Apoyo social ${ }^{*}$ & $3,1 \pm 1,0$ & $2,7 \pm 1,0$ & 0,772 \\
\hline Calidad de vida* & $4,3 \pm 1,0$ & $3,7 \pm 0,9$ & 0,479 \\
\hline
\end{tabular}

DE: Desviación estándar.

* Puntaje COOWONCA $\left({ }^{13}\right)$.
Tabla 7. Hospitalizaciones por reacciones adversas medicamentosas en los pacientes con prescripción de medicación potencialmente inadecuada o interacción droga-enfermedad potencialmente adversa.

\begin{tabular}{lc}
\hline Reacción adversa medicamentosa & $\begin{array}{c}\text { Frecuencia } \\
\mathrm{n}=115\end{array}$ \\
\hline $\begin{array}{l}\text { Hemorragia digestiva alta inducida por AINEs } \\
\text { Hipoglicemia inducida por glibenclamida } \\
\text { (en pacientes con betabloqueadores) }\end{array}$ & 13 \\
$\begin{array}{l}\text { Intoxicación digitálica } \\
\text { Síndrome caídas inducido por diazepam } \\
\text { (con fractura de cadera) }\end{array}$ & 7 \\
$\begin{array}{l}\text { Bloque AV 3er grado } \\
\text { Hemorragia digestiva por aspirina } \\
\text { dosis }>325 \text { mg/día }\end{array}$ & 3 \\
Total (\%) & 1 \\
\end{tabular}

Tabla 7 muestra las hospitalizaciones por reacciones adversas medicamentosa en los pacientes con prescripción de medicación potencialmente inadecuada o interacción drogaenfermedad potencialmente adversa.

\section{DISCUSIÓN}

La prevalencia de prescripción de medicación potencialmente inadecuada en los pacientes hospitalizados al momento de su internamiento fue de $12,4 \%$, siendo mucho menor la prevalencia hallada durante la hospitalización y al alta. Se encontró asociación directa entre la prescripción de medicación potencialmente inadecuada o interacción droga-enfermedad potencialmente adversa con el número de fármacos, número de enfermedades y la calificación del dolor en el puntaje de COOP/WONCA para calificar la calidad de vida y evaluación funcional de la salud. Por otro lado, la prescripción de medicación potencialmente inadecuada o interacción drogaenfermedad potencialmente adversa detectada al ingreso hospitalario se relacionó significativamente con la hospitalización por reacción adversa medicamentosa, siendo esta última la causa directa del internamiento en un $26,1 \%$ de los casos. 
La prevalencia de prescripción potencialmente inadecuada de $12,4 \%$ al ingreso hospitalario no es muy diferente de otros estudios. Chin y col. encontraron una prevalencia de $10,6 \%$ en pacientes mayores de 65 años al ingreso a un servicio de Emergencia $\left({ }^{10}\right)$; durante la hospitalización y al alta del paciente, las prevalencias fueron $3,6 \%$ y $5,6 \%$, respectivamente; en nuestro estudio fueron $3,4 \%$ y $2,1 \%$ en relación a las mismas condiciones. Existe otro estudio de Onde y col. $\left({ }^{14}\right)$, cuyo objetivo fue la prevalencia intrahospitalaria únicamente, encontrando que $14,6 \%$ de los pacientes internados en un grupo de hospitales universitarios italianos tenía al menos un medicamento prescrito inapropiadamente. La mayor parte de estudios de prevalencia de este problema está relacionada a adultos mayores de consultorios externos, nurserías e indigentes (homeless). Liu y col. realizaron un estudio metaanalítico sobre los principales estudios publicados hasta el 2001. De los 11 estudios analizados, la prevalencia osciló entre $40 \%$ en nurserías y $21,3 \%$ en pacientes adultos mayores ambulatorios $\left({ }^{15}\right)$. De acuerdo a estos estudios, la prevalencia encontrada en nuestro estudio sería baja. Uno de los factores para esta incidencia relativamente baja podría ser la existencia en EsSalud de un petitorio farmacológico $\left({ }^{16}\right)$ que excluye de antemano los siguientes fármacos incluidos en la lista de Beers de prescripción inadecuada: propoxifeno, fenilbutazona, pentazocina, trimetobenzamide, doxepin, meprobamamto, clorzoxazona, ciclobenzaprina, oxibutinina, ciclandelato, dipiridamol, mesilatos de ergot (co-dergocrina) y ticlopidina. Al momento, no existen estudios nacionales sobre este tópico.

En el presente estudio, los fármacos más frecuentemente prescritos inadecuadamente antes de ser hospitalizados fueron diazepam, digoxina (dosis superiores a $0,125 \mathrm{mg} /$ día), hierro (dosis superiores a $325 \mathrm{mg} /$ día), clorfeniramina y amitriptilina. En los estudios de Chin y col. $\left({ }^{10}\right)$, fueron difenhidramina, indometacina, meperidina y ciclobenzaprina; en las investigaciones de Onder y col. $\left({ }^{14}\right)$, la frecuencia de fármacos fueron ticlopidina, digoxina, amitriptilina, clordiazepoxido y diazepam; finalmente, en los estudios metaanalíticos de Liu col., figuran propoxifeno, amitriptilina, benzodiazepinas (diazepam, flurazepam) y dipiridamol $\left({ }^{15}\right)$.

La prevalencia de interacción droga-enfermedad potencialmente adversa al ingreso, durante la hospitalización y al alta fue $13,4 \%, 5,4 \%$ y $4,2 \%$, respectivamente. Comparativamente, otros estudios reportan sólo $5 \%, 0,6 \%$ y $1,2 \%$ en los tres casos señalados anteriormente, aunque este último estudio fue en pacientes ancianos en un servicio de Emergencia $\left({ }^{10}\right)$. En nuestro estudio, las interacciones droga enfermedad potencialmente adversa más frecuentes fueron úlcera péptica con antiinflamatorios no esteroideos, enfermedad pulmonar obstructiva crónica con beta bloqueadores y sedantes, diabetes mellitus y beta bloqueadores (en pacientes con hipoglicemiantes orales) y, finalmente, hipertrofia benigna de próstata con amitriptilina y clorfeniramina. En el estudio de Chin y col., los trastornos de la coagulación relacionados con anticoagulación y la enfermedad obstructiva crónica fueron las más frecuentes; ambos interaccionaron con beta bloqueadores, antiinflamatorios no esteroides, aspirina y sedantes-hipnóticos $\left({ }^{10}\right)$. Golderber y col. $\left({ }^{17}\right)$ publicaron que hasta un $21,5 \%$ tenía este problema relacionado con medicamentos. Sin embargo, debe anotarse que la población estudiada por estos autores fue de pacientes considerados de alto riesgo, tomando más de 3 medicamentos y mayores de 50 años de edad.

El antecedente de múltiples fármacos y la polipatología fueron los más consistentes predictores de prescripción de medicación potencialmente inadecuada o interacción drogaenfermedad potencialmente adversa. La polifarmacia en sí misma es un factor muy relacionado con la alta incidencia de reacciones adversas medicamentosas $\left({ }^{18}\right)$. Otro de los factores predictores del problema en estudio 
fue el ítem dolor del puntaje de COOP/ WONCA. El manejo del dolor crónico, según los estudios de Bernabei y col., es actualmente inadecuado, especialmente en los ancianos con neoplasias $\left({ }^{19}\right)$.

De los resultados obtenidos en el presente estudio es posible hacer algunas recomendaciones para la prevención de la prescripción de medicación potencialmente inadecuada e interacción droga-enfermedad potencialmente adversa, como son: incentivar el uso racional de fármacos en el anciano, difundir los criterios explícitos de Beers sobre prescripción potencialmente inadecuada y la educación sobre aspectos importantes de la administración de fármacos en ancianos, especialmente tópicos sobre terapia del dolor en este grupo etáreo.

Existen algunas limitaciones en el presente estudio. Una de la principales es que los hallazgos no son posibles de generalizar para otros hospitales, ya sea de la seguridad social ni los pertenecientes al Ministerios de Salud. El Hospital Almenara es un hospital de alta complejidad y de IV nivel, donde se recibe transferencias de los diferentes centros hospitalarios periféricos de la red. La realidad hospitalaria difiere significativamente de otros centros hospitalarios de menor nivel de EsSalud. Se hace necesario estudios más amplios que comprometan a hospitales nivel III y II de Lima y del interior del país, para conocer la verdadera dimensión del problema de prescripción de medicación potencialmente inadecuada e interacción droga-enfermedad potencialmente adversa en el adulto mayor. Por otro lado, hacen falta investigaciones que incluyan el seguimiento de los pacientes después del alta, para observar la influencia sobre la calidad de vida, morbilidad y mortalidad de la prescripción de medicación potencialmente inadecuada e interacción drogaenfermedad potencialmente adversa.

En conclusión, el presente estudio sobre el uso potencialmente inadecuado de medicamentos en adultos mayores internados en el Hospital Nacional Guillermo Almenara Irigoyen, en Lima, Perú, se detectó la prescripción de uno o más medicamentos inapropiados al ingreso, durante la hospitalización y al alta en $12,4 \%, 3,4 \%$ y $2,1 \%$, respectivamente. Los cinco medicamentos más frecuentemente prescritos inadecuadamente antes de ser hospitalizados fueron diazepam, digoxina (dosis superiores a $0,125 \mathrm{mg} /$ día), hierro (dosis superiores a $325 \mathrm{mg} /$ día), clorfeniramina y amitriptilina. Durante la hospitalización, los fármacos más frecuentes fueron diazepam e hioscina, aunque la prevalencia de prescripción inadecuada fue mucho menor. Se observó interacción droga-enfermedad potencialmente adversa al ingreso, durante el internamiento y al alta en porcentajes de $13,4 \%, 5,4 \%$ y $4,2 \%$, respectivamente. Las cinco interacciones drogaenfermedad potencialmente adversas más comunes fueron úlcera péptica con antiinflamatorios no esteroideos, enfermedad pulmonar obstructiva crónica con beta bloqueadores y sedantes, diabetes mellitus y betabloqueadores (en pacientes con hipoglicemiantes orales) y, finalmente, hipertrofia benigna de próstata con amitriptilina y clorfeniramina. Se recomienda la realización de más estudios sobre este tópico, idealmente a nivel nacional, para conocer los patrones; $y$, con estos datos, diseñar programas de intervención para evitar la prescripción inadecuada de fármacos en el adulto mayor.

\section{AGRADECIMIENTOS}

A María Xesús Froján Parga (PhD) de la Universidad Autónoma de Madrid, España por su excelente asesoría a la presente investigación.

A EsSalud por la Subvención de Proyectos de Investigación 2002, Resolución $\mathrm{N}^{\circ}$ 362-GCRHEsSalud-2002 de la Gerencia Central de Capacitación y Desarrollo, Gerencia de Recursos Humanos. Asimismo, al Dr. Gerardo Lira Linares, a los médicos asistentes y residentes (período 20022003) del Servicio de Geriatría del Hospital 
Almenara. Al Dr. Demetrio Molero Jefe del servicio de Farmacovigilancia del Hospital Almenara, por su revisión y sugerencias.

\section{REFERENCIAS BIBLIOGRÁFICAS}

1. Aparasu RR, Mort JR. Inappropriate prescribing for the elderly: Beers criteria-based review. Ann Pharmacother. 2000;34:338-46.

2. Piecoro LT, Browning ST, Prince TS, Scott T, Scutchfield FD. A database analysis of potentially inappropriate drug use in an elderly Medicaid population. Pharmacotherapy. 2000;20:221-8.

3. Beers MH, Ouslander JG, Rollinger I, Reuben DB, Brooks J, Beck JC. Explicit criteria for determining inappropriate medication use in nursing home residents. Arch Intern Med. 1991;151:1825-32.

4. Willcox SM, Himmelsten DU, Woolhandler S. Inappropriate drug prescribing for the community-dwelling elderly. JAMA. 1994:272:292-6.

5. Beers MH. Explicit criteria for determining potentially inappropriate medication use by the elderly. Arch Intern Med. 1997;157:1531-6.

6. Hanlon JT, Shimp LA, Semla TP. Recent advances in geriatrics: drug-related problems in the elderly. Ann Pharmacother. 2000;34:360-5.

7. Stuck AE, Beers MH, Steiner A, Aronow HU, Rubenstein LZ, Beck JC. Inappropriate medication use in communityresiding older persons. Arch Intern Med. 1994;154:2195200.

8. Spore DL. Mor V, Larrat P, Hawes C, Hiris J. Inappropriate drug prescription for elderly residents of board and care facilities. Am J Public Health. 1997;87:4074-9.

9. Aparasu RR, Fliginger SE. Inappropriate medication prescribing for the elderly by office-based physician. Ann Pharmacother. 1997;31:823-9.

10. Chin MH, Wang LC, Jin L, Mulliken R, Walter J, Hayley DC, et al. Appropriateness of medication selection for older persons in an urban academic emergency department. Academic Emergency Medicine. 1999;6:1232-42.
11. Folstein M, McHugh P. «Mini-mental state», a practical method for grading the cognitive state of patients for the clinician. J Psychiat Res. 1975;12:189-98.

12. Katz S, Ford AB, Moskowitz RW, Jackson BA, Jaffe MW. Studies of illness in the aged. The index of ADL: A standardized measure of biological and psychosocial function. JAMA. 1963;185:914-19.

13. van Weel C, König-Zahn C, Touw-Otten FWMM, van Duijn NP, Meyboom de Jong B. (trad.) Measuring functional health status with the COOP7WONCA charts. A manual. Groningen: Noordelijke Centrum voor Gezondheidsvraagstukken (ncg)/ Northern Centre of Health Care Research (nch); 1995.

14. Onder G, Landi F, Cesari M, Gambassi G, Carbonin P, Bernabei R. Investigators of the GIFA Study. Inappropriate medication use among hospitalized older adults in Italy: results from the Italian Group of Pharmacoepidemiology in the Elderly. Eur J Clin Pharmacol. 2003;59(2):157-62.

15. Liu GG, Christensen DB. The continuing challenge of inappropriate prescribing in the elderly: an update of the evidence. J Am Pharm Assoc. 2002;42:847-57.

16. Comité Farmacológico Nacional, EsSalud. Petitorio Farmacológico Nacional EsSalud. Lima: EsSalud; 2000.

17. Goldberg RM, Mabee J, Chan L, Wong S. Drug-drug and drug-disease interactions in the ED: analysis of a high-risk population. Am J Emerg Med. 1996;14(5):447-50.

18. Bjerrum L, Sogaard J, Hallas J, Kragstrup J. Polypharmacy: correlations with sex, age and drug regimen. Eur J Clin Pharmacol. 1998;54:197-202.

19. Bernabei R, Gambassi G, Lapane K, Landi F, Gatsonis C, Dunlop R, et al. Management of pain in elderly patients with cancer. JAMA. 1998;279:1877-82.

Manuscrito recibido el 09 de febrero de 2005 y aceptado para publicación el 15 marzo 2005.

Correspondencia: Dr. Teodoro J. Oscanoa Espinoza

Calle Jorge Muelle 433 Dpto 806, San Borja. Lima 41, Perú

Correo-e: teodorojoscanoa@yahoo.com

toscanoae@sanfer.unmsm.edu.pe 